\title{
Estudio sobre Actitudes y Comportamientos que influyen en la Masculinidad desde la Percepción de Estudiantes de la Universidad Nacional de Asunción (Paraguay)
}

\author{
Study on Attitudes and Compartments that influence Masculinity from \\ the Perception of Students of the U.N.A (Paraguay)
}

\author{
Valerio Olguín Carvallo \\ Univ. Nacional de Asunción \\ valerioolguincarvallo@gmail.com \\ ORCID https://orcid.org/0000-0003-2889-554X
}

\section{Resumen}

El presente artículo expone los resultados de un estudio de grado llevado a cabo en el año 2016 cuyo objetivo apuntó a analizar las características de la masculinidad tradicional desde la percepción de las y los estudiantes de Psicología, Trabajo Social, Derecho y Medicina de la Universidad Nacional de Asunción en el año 2016, para lo cual se procedió a identificar la influencia del sistema patriarcal en la configuración de las actitudes y comportamientos en los siguientes ámbitos: las responsabilidad que asumen varones y mujeres en la sexualidad y la salud reproductiva; la paternidad y la violencia de género en el año 2016. El diseño metodológico, es de tipo exploratorio descriptivo con un enfoque cualitativo en vista a que para la recolección de datos se utilizó como instrumento la entrevista semiestructurada abierta y cerrada con un cuestionario guía compuesto de 30 preguntas. La muestra estuvo compuesta de 24 entrevistas a estudiantes ( 12 varones y 12 mujeres) de los últimos años de las carreras de edades entre 23 a 45 años residentes en la ciudad de Asunción y el departamento central, las mismas corresponden a estudiantes de las carreras de Psicología, Trabajo Social, Derecho y Medicina.

Palabras clave: Roles de género; masculinidad hegemónica; masculinidades; paternidad; sexualidad y salud reproductiva. 


\begin{abstract}
This article presents the results of a degree study carried out in 2016 whose objective aims to analyze the characteristics of traditional masculinity from the perception of the students of Psychology, Social Work, Law and Medicine of the National University of Asunción in 2016, for which we proceeded to identify the influence of the patriarchal system in the configuration of attitudes and behaviours in the following areas: the responsibilities assumed by men and women in sexuality and reproductive health; paternity and gender violence in 2016. The methodological design is of a descriptive exploratory type with a qualitative approach in view of the fact that the open and closed semi-structured interview was used as an instrument for data collection, with a guiding questionnaire composed of 30 questions. The sample consisted of 24 interviews with students (12 men and 12 women) of the last years of the careers aged between 23 to 45 years residing in the city of Asunción and the central department, they correspond to students of the careers of Psychology, Social Work, Law and Medicine.
\end{abstract}

Keywords: Gender roles; hegemonic masculinity; masculinities; fatherhood, sexuality and reproductive health. 


\section{Introducción}

A continuación, se presenta una general y breve descripción de las perspectivas teóricas, la matriz de análisis e indicadores que se tuvieron en cuenta para el desarrollo y la aplicación del estudio sobre las características de la masculinidad tradicional desde la percepción de las y los estudiantes de la Universidad Nacional de Asunción (Paraguay).

En primer lugar, como parte de un recorrido histórico de las perspectivas teóricas sobre masculinidades se menciona el surgimiento de los Men's Studies, resaltando la gran influencia que este y las ciencias sociales han recibido de los movimientos feministas y los estudios de género. El objetivo de este apartado consiste en realizar un acercamiento a las principales corrientes que se han dedicado al estudio de las masculinidades representado en sus inicios principalmente por países anglosajones (EE.UU., Australia, Canadá y Reino Unido) para posteriormente citar las corrientes y perspectivas latinas que se encargan de investigar sobre masculinidades.

Posteriormente, se exponen tres escenarios de construcción y socialización masculinas, la razón de ubicar en tres escenarios los estudios sobre masculinidades apunta a que el lector/a logre identificar ciertos patrones que tienen en común dichos estudios, que, a pesar de haber sido realizados en contextos y regiones diferentes poseen patrones similares en cuanto a la producción y reproducción de espacios de socialización y legitimación social de las masculinidades. Siendo el espacio público, junto a las instituciones modernas como la familia y la escuela, los escenarios por excelencia que promueven la construcción y socialización de la identidad masculina mediante la homofobia y la naturalización de la violencia.

En segundo lugar, en lo respecta al apartado dos se presenta la matriz de análisis utilizada en este estudio, con el fin de identificar la influencia del sistema patriarcal en estudiantes universitarios, conocer los cambios en las actitudes y comportamientos de varones y mujeres en las nuevas generaciones. En este mismo apartado se describe el marco teórico utilizado para el análisis sobre la masculinidad tradicional y hegemónica, desde la percepción de autores como Connell (1995) Kimmel (1997) Viveros Vigoya (2003) para estos los términos "masculinidad tradicional y Masculinidad Hegemónica" son sinónimos, la primera, engloba a los roles que una sociedad en particular atribuye o espera que sean realizadas por los varones (proveedor, protección, etc.) y la segunda, define un tipo de relación asimétrica entre los géneros y otros tipos de masculinidades. Para este estudio sobre las masculinidades desde la percepción de estudiantes se tuvieron en cuenta ámbitos como: familia, escuela, sexualidad, salud reproductiva, paternidad y la violencia. La investigación se realizó entre hombres y mujeres, partiendo de que todos/as formamos parte del mismo tejido social que reproduce el sistema patriarcal.

En el tercer y último apartado se presenta el análisis de los datos y los principales resultados del estudio mediante algunos gráficos. Esta sección se divide en 6 indicadores de estudio. Entre los resultados se destaca que estudiantes, tanto varones y mujeres, identifican y perciben la influencia del sistema de la división sexual del trabajo a través de las tareas domésticas y mediante los mandatos del binarismo genérico imperante en la sociedad paraguaya. En lo que respecta a actitudes y comportamientos considerados negativos sobre los roles de género de cada individuo se concluyó que reproducir actitudes y comportamientos machistas es más frecuente en los varones. Dicha conclusión se da luego de constatar que determinadas tareas y comportamientos vinculados con el cuidado y la salud reproductiva siguen siendo exclusiva responsabilidad de las mujeres, situación que reafirma que la influencia negativa de los roles tradicionales de género sin duda trasciende el nivel académico, profesional, político y social. 


\section{Contexto General de los Men's Studies}

A pesar del esfuerzo de sectores religiosos y políticos en desmeritar el aporte de los estudios feministas para las ciencias sociales, el género como categoría analítica se ha convertido en un campo de saber especializado que transversaliza cualquier disciplina y teoría. Si bien, en algún momento histórico estuvo restringido al estudio de las mujeres, en los años 80, este movimiento social, político y académico abordó el ámbito de las masculinidades y luego se extendió en todas las áreas.

En cuanto a los estudios sobre las masculinidades este se desarrolló principalmente en países anglosajones (EE.UU., Australia, Canadá y Reino Unido) bajo el nombre de Men's studies (Rubio, 2001, p. 2), de manera que a partir de este campo-Men's studies- se comienza a tener en cuenta la idea de un modelo hegemónico de masculinidad que jerarquiza a aquellas -masculinidades- que no cumplen o reúnen los valores que identifican al modelo hegemónico. Esto nos lleva a la idea de que existen una diversidad de expresiones masculinas que se encuentran atravesadas por las categorías como la raza, clase social y el género y la diferencia generacional, sin embargo, estas varían según los tiempos y lugares.

Entre los modelos teóricos más utilizados para pensar y explicar la articulación de sistemas de opresión (raza, clase y género) se encuentra la interseccionalidad" "como un enfoque y una perspectiva que busca comprender el funcionamiento de las opresiones entrelazadas como una matriz de dominación", en la cual no existen categorías de poder jerarquizadas o sumadas, sino ejes de poder entretejidos, que configuran redes de posiciones sociales estructuradas por la inseparabilidad de las categorías de género, raza, clase, sexualidad, edad, capacidad, entre otras categorías de diferencia (Viveros y Gregorio, 2014, p. 10-11).

Continuando con contribuciones de los Men's studies según Rubio (2001) los mismos se dan en el ámbito de los estudios empíricos, ante todo, porque ofrecen descripciones de las múltiples prácticas y concepciones relacionadas con la masculinidad que caracterizan o han caracterizado cada sociedad y cada época, por ejemplo:

"el obrero inglés, centra su masculinidad en la alta valoración del trabajo manual, el desdén por la actividad intelectual y un marcado sexismo, mientras que la masculinidad en la burguesía se define alrededor del éxito en actividades intelectuales, comerciales y/o empresariales, y tiene como uno de sus valores más distinguidos el trato 'exquisito' a las mujeres." (Rubio, 2001, p. 2).

También puede suceder, que los imperativos morales de la masculinidad consisten siempre en que el hombre ha de ejercer de proveedor, preñador y protector, pero asumen formas y contenidos culturales tan dispares en cada lugar, época o estrato social que no se puede hablar de una

\footnotetext{
${ }^{1}$ Dicho enfoque no es novedoso dentro del feminismo, pero sí en variados contextos académicos y políticos. En sus inicios, los estudios de interseccionalidad se dirigieron contra la hegemonía del feminismo blanco, demostrando —como lo hicieron el grupo pionero del Black feminism, la Colectiva del Río Combahee, en 1977, o académicas feministas como Angela Davis, Audre Lorde, bell hooks, June Jordan, Patricia Hill Collins, María Lugones- que la categoría "mujer", empleada por muchas teorías feministas, había sido constituida basándose en la experiencia de las mujeres privilegiadas por razones de clase y raza, ignorando la situación de las mujeres cuya situación social era diferente.
}

Revista del Laboratorio Iberoamericano para el Estudio Sociohistórico de las Sexualidades https://doi.org/10.46661/relies.XXXX 
masculinidad universal (Rubio, 2001, p. 2).

Ahora bien, en un contexto no eurocéntrico los denominados Men's studies también han demostrado cómo los mandatos de la masculinidad y la feminidad -coercitivamente heredadosafectan la salud y la educación de hombres y mujeres en distintos niveles y formas (Nascimento, 2015, p. 14-16). Sobre todo, cuando lo masculino se define como:

"un modelo o la idea de un hombre fuerte, varonil, profesional, hombre de familia, del mundo público, de la calle, competitivo, con poca conexión con la esfera de la vida privada, alejado del mundo de los afectos, con un distanciamiento de las emociones, y que puede comportarse de manera agresiva (e incluso violenta) contra las mujeres y hombres" (Nascimento, 2011, p. 14-16).

Si bien es cierto que cada cultura construye un modelo masculino y femenino propio de los procesos de socialización cultural, no podemos obviar que los valores y patrones culturales dominantes en países como Paraguay están muy vinculados a los procesos neocoloniales. Es decir que, además de (re)producir un sistema sociopolítico que establece roles y jerarquías mediante el sexo, el color de piel, la edad, la clase, los individuos también debemos competir por cumplir criterios de civilización y desarrollo que estamos obligados a alcanzar para ser aceptados socialmente.

Para Alexandra Kollontai (1976) lo que se observa de fondo es el sistema patriarcal y la división sexual del trabajo que ha perpetuado el sometimiento, subordinación y opresión de los hombres hacia las mujeres. Según Kollontai (1976) los hombres no son buenos o malos por naturaleza, sino que su dominio responde al desarrollo histórico que tiene como base la propiedad privada, el sometimiento de la naturaleza y el cuerpo de las mujeres.

Es por esto que resulta importante tener en cuenta que, para abordar y entender la masculinidad, primero debemos entender los significados que le atribuye cada sociedad y lo que esta define como normativo, bueno, ordenado, y recomendable para los varones; así como lo que se considera inadecuado, desordenado o abominable (Guasch, 2008, p. 38-39). Esto último, en vista que el estudio de las masculinidades incluye a los sujetos masculinos que transgreden la normatividad genérica de los roles binarios varón-mujer.

\subsection{Escenarios de Construcción y Socialización Masculinas}

A continuación, se presentan tres estudios realizados en diferentes países, pero con un factor común en los mismos, el estudio de las masculinidades y los espacios de reproducción, socialización y legitimación social. Al decir de Guevara (2006) el primer espacio de construcción y socialización de lo masculino implica a dos instituciones modernas como la familia y la escuela, que se constituyen en escenarios por excelencia de legitimación y aceptación social de cada contexto. A esto se suma Barbero (2017) -segundo escenario- que coincide con Guevara (2006) respecto de la necesidad de "los espacios públicos" como parte del proceso de socialización de la identidad masculina que es construida en oposición a la feminidad, la homofobia, y la naturalización de la violencia.

Por último y de modo a realizar un acercamiento a las características de la(s) masculinidad(es) paraguayas se exponen en el tercer espacio o espacios de socialización y legitimación identificado y diferenciado por los adolescentes participantes del estudio realizado por Negrete (2016); estos espacios se dividen en dos: el privado que juega un papel fundamental en la conformación de lo masculino (familia) y el espacio público donde esta masculinidad es probada, fortalecida y consolidada (el colegio y/o la calle), en la interacción entre pares. Mediante esta diferenciación entre lo privado y lo público Negrete (2016) presenta tres tipologías generales de masculinidad: la hegemónica, la subordinada o altruista y la flexible. 


\section{1.a Primer escenario: La Familia y la Escuela}

A nivel general, en los últimos años se ha hecho mención de los cambios y el surgimiento de "nuevas masculinidades" debido a cuestiones relacionadas con: la participación de hombres en las tareas del hogar o trabajo doméstico y en el cuidado de los niños, niñas y enfermos, o por la demostración pública de sus sentimientos, al respecto Guevara (2006) sostiene que estos no representan cambios significativos "sino que en realidad lo que existe es una ligera modificación a un modelo light de ejercicio del poder que opera a través un machismo invisible o moderado que se oculta tras nuevas y viejas prácticas" (Cruz 2004; Minello 2005).

Según Guevara (2006) este modelo light sustentó la modificación de algunas prácticas y discursos masculinos que en realidad no responden a una transformación consciente de los sujetos en cuestión, sino que son producto de "procesos psicosociales que involucran estructuras institucionales y condiciones personales que hacen posible la emergencia de nuevos referentes sociales de lo masculino" (Ariza y Oliveira, 2004 en Guevara, 2006, pp. 3), lo que significa que la supuesta modificación de los roles masculinos podría deberse a cuestiones propias de cada contexto individual, social o económico antes que a una pretendida igualdad entre hombres y mujeres.

Si partimos de la idea de que "la construcción de la masculinidad es socializada en diferentes escenarios sociales donde las capacidades y actividades masculinas se significan en función del estatus y el poder que le otorgan" (Guevara, 2006, pp. 5) los sujetos masculinos y femeninos. Podría decirse que efectivamente es en el espacio público donde se construye la identidad masculina mediante tres factores; "el sentido de pertenencia a un grupo social; los atributos derivados de la posición que ocupa ese grupo en la sociedad; y el sentido del Yo producto de la historia colectiva y de la propia biografía personal" (pp. 6).

Estos tres factores sumados a la clase, etnia, edad o sector religioso al que pertenece un sujeto definen en gran medida la existencia no de una sino de "múltiples identidades masculinas" (Guevara, 2006). No obstante, son dos "las instituciones modernas" que se han constituido como los pilares para la socialización y legitimación de la masculinidad hegemónica "la familia y la escuela", a través de estas, y de "sus sistemas normativos, prácticas y discursos se reproducen y recrean los mandatos simbólicos de lo que significa ser un hombre y una mujer" (Guevara, 2006, pp. 2). Sin embargo, lo que nos interesa del aporte de Guevara (2006) es la existencia de una masculinidad hegemónica y sus mandatos que excluye a aquellos varones que no cumplen con aquello que de forma simbólica implica ser hombre.

\section{1.b Segundo escenario: El rol de las Instituciones educativas en la reproducción de patrones culturales}

En este segundo escenario se presenta un estudio llevado a cabo en entre los años 2013 y 2014 en centros educativos españoles donde se investigó el papel que juega la homofobia como una forma específica de violencia en la construcción, reproducción y control de la masculinidad entre los hombres jóvenes de estudiantes del nivel primario y secundario. Para la recolección de datos se utilizó un método mixto donde se aplicaron 3236 cuestionarios por alumnado de $\mathrm{ESO}^{2}$, bachillerato, FP y PCPI y 250 cuestionarios de profesorado de todos los niveles educativos. En una primera etapa se utilizó como técnica la observación participante ${ }^{3}$ para la recolección de datos (cualitativa), para

\footnotetext{
${ }^{2}$ La Educación Secundaria Obligatoria (ESO) abarca a estudiantes de entre 12 a 16 años, mientras que en el bachillerato se estiman edades de entre 16 y 18 años. En cuanto al Programas de Cualificación Profesional Inicial (PCPI) están destinados a jóvenes entre 16 y 21 años que no hayan conseguido el título de ESO.

${ }^{3}$ En 47 talleres sobre diversidad sexual y familiar impartidos en 6 centros de educación infantil y primaria, así como 11 entrevistas en profundidad al profesorado, alumnado y familiares recogiendo sus experiencias relativas a la diversidad sexual y familiar en las aulas.
}

Revista del Laboratorio Iberoamericano para el Estudio Sociohistórico de las Sexualidades https://doi.org/10.46661/relies.5521 
luego realizar entrevistas a profundidad a jóvenes lesbianas, gais, bisexuales, transexuales (LGBT) y asexuales, donde relatan sus experiencias.

Entre los resultados se resalta que el acoso escolar es una realidad incesante y entre los principales motivos de burla, insulto y exclusión pueden distinguirse dos grupos:

"el primero, de donde se concluyó que las primeras burlas e insultos hacia los y las compañeras no estarían vinculadas al género ni la sexualidad, sino que giran en torno a las cuestiones físicas relativas al peso y la altura. Las experiencias del profesorado coinciden que es precisamente en los primeros años de la etapa de educación primaria en la que aparecen insultos como "mariquita" o "marimacho" dirigidos a aquellos niños que "hacen cosas de niñas" y viceversa (Barbero, 2017, p, 15).

Mientras que el segundo grupo, está relacionado a la segunda etapa del estudio y corresponde a los alumnos/os del nivel secundario, aquí los resultados revelaron que 8 de cada 10 estudiantes han presenciado burlas e insultos relativos a la orientación sexual y la identidad de género. No obstante, tanto el alumnado como el profesorado afirmó que la convivencia educativa es tranquila, a pesar de que sólo 1 de cada 10 participantes refirió no haber presenciado burlas, insultos, exclusión, amenazas o agresiones en su centro educativo (Barbero, 2017, p. 7).

La explicación que Barbero (2017) ofrece a dicha contradicción va en un apartado titulado "el acoso escolar como un juego de hombres", en dicho apartado éste refiere que los hombres que habían afirmado acosar constantemente a sus compañero/as duplican en cantidad a las chicas, y, esto se debe a que social y culturalmente, exaltamos un tipo de masculinidad que influencia poderosamente la vida de los hombres (Barbero, 2016, p. 13). ${ }^{4}$

En este sentido los resultados señalaron "tres lugares de riesgo en los centros educativos en lo relativo al acoso escolar: el momento del cambio de clases, el patio de recreo y durante las clases", por tanto, se concluyó que "el acoso escolar no tiene lugar en espacios privados o reservados, sino que sucede sobre todo en espacios donde el resto del alumnado está presente" (Barbero, 2017, p. 13).

Al presentar al acoso escolar como "un juego de hombres" Barbero (2017) los ubica como actores que perpetran y a la vez sufren la violencia como parte del proceso de socialización y legitimación de la identidad masculina, para lo cual se utilizan mecanismos como la confrontación, la negación a la feminidad y la homofobia como lo opuesto a la masculinidad (Barbero, 2017, p. 12). Es decir que: "la homofobia es el mecanismo por el cual se censura cualquier atisbo de feminidad en otros hombres ya que es la identidad masculina -y no la femenina- la que se construye en el temor a ser o parecer homosexual" (Kimmel, 1997 en Barbero, 2017, p. 17).

Según Barbero (2017) el hecho de que un gran porcentaje de alumnos varones heterosexuales sufren acoso por homofobia en los centros educativos confirmar lo señalado por diversos autores es decir que "la homofobia no se dirige exclusivamente contra las personas LGBT" (Leverenz, 1986; Blumenfeld, 1992; Parker, 1996; Kimmel, 1997; Borrillo, 2001; Pichardo, 2009, Barbero, 2017).

De ahí que este concluye que "la invisibilización y normalización de la homofobia y de la violencia entre pares encuentra su intersección en las relaciones de dominación/subordinación de las masculinidades, donde los hombres jóvenes se disputan constantemente midiendo los límites

\footnotetext{
${ }^{4}$ Es por ello que en el acoso escolar encontramos que los hombres jóvenes dirigen las burlas e insultos contra sus compañeras mujeres (40\%), pero incluso en mayor medida contra sus propios compañeros varones $(62,59 \%)$.
} 
masculinos con violencia, pero también con miedo y silencio" (Barbero, 2017, p. 22). ${ }^{5}$ Como se puede constatar la normalización y la naturalización del acoso escolar entre jóvenes, de tal forma que no se percibe como un tipo de violencia los insultos y burlas, ya que es "una forma amistosa de hablar y no hay que tomarlos como algo negativo".

\section{1.c Tercer escenario: las masculinidades y la violencia en adolescentes escolarizados de Asunción}

En un escenario nacional o local, en el año 2016 CLACSO $^{6}$ publicó un artículo producto de un estudio exploratorio-descriptivo sobre la caracterización de los discursos relacionados a las masculinidades en un grupo de adolescentes varones escolarizados en la ciudad de Asunción, Paraguay. El mismo tuvo como objetivos un acercamiento a los procesos relacionados a la construcción, consolidación y expresión de las masculinidades desde las perspectivas de los entrevistados, así como analizar el rol que juega el poder y la violencia en este proceso de construcción identitario. La muestra fue de 40 adolescentes entrevistados a través de grupos focales.

A fin de exponer los resultados de mayor relevancia para el este artículo en primer lugar se realizará una descripción general de los conceptos teóricos utilizados por el investigador ${ }^{7}$ para describir y analizar las masculinidades, y en segundo lugar se procederá a describir los resultados obtenidos que serán agrupados en dos categorías relacionales para la construcción y socialización de las masculinidades adolescentes en la familia y el colegio.

En un inicio Negrete (2016) parte de que la "masculinidad" no sería una categoría única con una definición con límites claros y de aplicación universal, sino que la masculinidad como categoría de estudio estaría modificada por el contexto cultural, social, económica y política donde ésta se inscribe, y a su vez, atravesada por variables como la edad, etnia, posición social entre otros (Badinter, 1992; Connell, 1998).

Por lo que para dicho estudio Negrete (2016) entiende que la denominada masculinidad "hegemónica" a decir de Connell (1995):

"La masculinidad hegemónica es la configuración de la práctica genérica que encarna la respuesta al problema de la legitimidad del patriarcado, es decir, es la que garantiza (o se toma para garantizar) la posición dominante de los hombres y la subordinación de las mujeres" (p.43).

Es decir, la masculinidad hegemónica tiene como base la relación de subordinación de determinadas masculinidades, así como la relación con las mujeres. En este caso, el género no sólo expresa los rasgos de una persona, sino que a través de un determinado proceso institucional y una dinámica de relaciones de poder define un orden social jerárquico, donde los hombres dominan sobre las mujeres y sobre otros hombres (Negrete, 2016, p. 7).

En este sentido, la masculinidad hegemónica necesita de un estatus de dominación sobre las demás "masculinidades", donde exista un consenso tácito que reconozca a esta masculinidad hegemónica

\footnotetext{
${ }^{5}$ Es por eso que uno de los participantes refirió que "los insultos referentes a la homosexualidad no se dicen en serio" y otro agregó que decir "maricón" a un amigo es "inofensivo", ya que la ofensa no radica concretamente en la orientación sexual, que puede ser "respetable" sino que simbólicamente decirlo es un desafío a la masculinidad. Esto en vista a que prácticamente la mitad $(49,60 \%)$ de los hombres jóvenes no cuenta a nadie que sufre acoso escolar, y en caso de pedir ayuda la proporción se invierte: las chicas prácticamente duplican a los chicos a la hora de contar las situaciones de acoso o buscar ayuda entre sus amistades y familiares

${ }^{6}$ Consejo Latinoamericano de Ciencias Sociales (CLACSO).

${ }^{7}$ Martin Negrete Psicólogo y Máster en Salud Pública. Investigador en temas de salud, sexualidad y género. Docente Universitario.
} 
como dominante de las otras. Dichas masculinidades son clasificadas por Connell (1997): la masculinidad subordinada que se sitúa alejada del ideal masculino tradicional, una suerte de masculinidades incompletas, inmaduras o relacionadas a "lo femenino", encarnada tradicionalmente en Occidente por la figura del hombre homosexual. La masculinidad marginada alude a aquellos hombres excluidos socialmente y que tienen acceso restringido al poder $y$ finalmente las masculinidades cómplices serían aquellas que si bien replican casi todos los patrones hegemónicos pero que no gozan de un alto estatus social y económico (Negrete, 2016, p.8).

De acuerdo con los resultados obtenidos por dicho estudio en su mayoría los adolescentes identificaron dos espacios diferenciados donde su identidad de género es desarrollada, uno privado que da origen a lo masculino (familia) y otro espacio público donde esta masculinidad es probada, fortalecida y consolidada (el colegio y/o la calle, y en ambos con la participación de los pares). Ahora bien, las ventajas percibidas de "ser hombre" para muchos de estos adolescentes se asocian a la capacidad productiva (ganar más dinero), la libertad y autonomía que se interpreta como mayor capacidad de control sobre el ambiente y las relaciones. Esta percepción de poseer o no ciertos atributos los motiva constantemente a evaluar su medio ambiente social, y de acuerdo a las circunstancias moldear su comportamiento negociando el uso del poder o cederlo para salvaguardar alguna posición en este orden social masculino (Negrete, 2016, p. 15-18).

Los atributos descritos por los adolescentes fueron agrupados en tres tipologías generales de masculinidades y estas cobran significado únicamente en el contexto físico y social en el que fueron mencionadas;

a) Masculinidad hegemónica: sus características serían la competencia, los deportes, solidez del grupo, amistad férrea y cómplice, uso de chistes y burlas como medio de control, uso explícito de la violencia como medio de coacción, poder y control. Atributos: aspecto físico, estatus social, violencia y control.

b) Masculinidad subordinada: altruismo carente de todos los atributos relacionados con la masculinidad hegemónica, lo cual la sitúa en las márgenes del orden social establecido. Sin embargo, como único atributo percibido como "positivo" por parte del modelo hegemónico y el cual sería un requisito para evitar la marginación total es el "altruismo". Esta categoría estaría conformada por los tranquilos, estudiosos, aislados, tímidos, los que evitan conflictos huyendo o a veces aliándose con los hegemónicos.

c) Masculinidad flexible: los adolescentes inscriptos en esta categoría se caracterizan por la inteligencia, buena relación con las chicas y profesores, y con la familia. Se vinculan más a actividades artísticas que a deportivas. Tienden a mostrar una actitud solidaria y a defender a los subordinados. Atributos: asertividad y competencia social.

A nivel discursivo, gran parte de los dichos populares y en especial los canticos de las hinchadas durante las competencias deportivas tiene un carácter netamente homoerótico-sexual: "Le rompimos el culo"; "Tenés que usar vaselina", "La tiene adentro"; "No se van a poder sentar". Se observó como estas expresiones si bien contienen un contenido abiertamente homoerótico/sexual, a nivel de la práctica, es decir, la función que cumple es abiertamente homofóbica ya que busca humillar, al denigrar y/o descalificar al oponente (Negrete, 2016, p. 22).

En otros casos, se observa la integración de compañeros abiertamente gay, pero cuya asimilación está dada por una negociación con el grupo dominante, donde se pacta una subordinación representada por el rol del "buen compañero" o el que "no molesta a nadie y el que ayuda siempre"; el que "está pendiente de todos". En este contexto, la masculinidad "altruista" y subordinada al grupo dominante es la que algunos varones gais asumirán como un medio de sobrevivencia en un ambiente social hostil. Por último, y como voces alternativas y disidentes están algunos pocos 
relatos que muestran una visión pluralista y respetuosa a la diversidad sexual.

En relación a las mujeres, la posición asumida resulta ambigua; por un lado, los textos analizados muestran contenidos misóginos, donde (1) se destaca la subordinación de la mujer en ciertos escenarios y contextos y una visión estereotipada de los roles femeninos, por otro lado, (2) una visión paternalista, que equipara lo femenino a lo infantil destacando rasgos emotivos que restan "madurez" a muchas de sus acciones. En este último caso, nótese la figura del varón como un complemento racional y pragmático necesario para la solución de problemas. Por último, en algunos casos se escuchan voces disidentes a estos discursos más tradicionales, donde (3) se valora la equidad en la diferencia.

En relación a la violencia hacia la mujer, ésta es enérgicamente rechazada y censurada por todos, sin excepción del tipo de grupo. Aquí nuevamente se define violencia hacia la mujer en función a la violencia física. Otros tipos de acciones de control, ejercicio del poder o subordinación no son percibidos como violencia, existiendo una ceguera a esa interpretación (Negrete, 2016, p. 24).

En resumen, Negrete (2016) refiere que los discursos masculinos vinculados a las mujeres y los homosexuales guardan una clara relación, y a nivel descriptivo poseen las mismas características. Los varones con construcciones de género hegemónicas poseen una mirada misógina y homofóbica, donde el uso disciplinador de la violencia física, psicológica y simbólica tiene un objetivo de sujeción y subordinación; por otro lado, los discursos aparentemente desprovistos de violencia, mantienen una postura paternalista, donde mujeres y homosexuales son vistos como sujetos inmaduros e imperfectos que demandan cuidado y control. Por tanto, ambos constituyen un factor de riesgo para el bienestar de las mujeres y de los mismos varones (p. 32).

En este punto se puede señalar que tanto Negrete (2016) como Barbero (2017) coinciden en dos escenarios principales de socialización de la masculinidad, por un lado, la familia (escenario privado/doméstico) y por otro el ámbito educativo (escenario público). Este último está estrechamente relacionado con la socialización del espacio y la legitimación de los tipos de masculinidades que estamos reproduciendo. En ambos estudios se exponen dos contextos educativos y regiones diferentes, pero escenarios muy parecidos para la consolidación de concepciones "esencialistas de lo masculino" en los cuales sobrevaloran los atributos como el poder físico, económico y simbólico, y, sobre todo, el uso de la violencia como necesarios para situarse al interior del orden jerárquico masculino.

En este punto se destaca la importancia de explorar los procesos de socialización de la masculinidad en estudiantes universitarios de Paraguay, sobre todo partiendo de que la educación universitaria debe promover y garantizar el ejercicio de derechos y la participación en igualdad de condiciones para todos y todas. Es por ello que conocer la percepción de este sector educativo nos invita a reflexionar y comprender sobre el rol de la universidad en cuanto a la reproducción de dichos patrones, y a cuestionarnos sobre los cambios a los cuales deberíamos apuntar si lo que se busca es construir una sociedad con participación social y política equitativa e incluyente.

Dicho lo anterior, este estudio buscó identificar las características de la masculinidad tradicional desde la percepción de las y los estudiantes de Psicología, Trabajo Social, Derecho y Medicina de la Universidad Nacional de Asunción en el año 2016, para esto, en primer lugar se procedió a identificar la influencia del sistema patriarcal en la configuración de las actitudes y comportamientos en los siguientes ámbitos: las responsabilidad que asumen varones y mujeres en la sexualidad y la salud reproductiva; la paternidad y la violencia por razón de género en el año 2016. 


\section{Marco teórico conceptual: matriz de análisis}

En primer lugar, se parte de una definición general de lo que se define como patriarcado, este término ha sido utilizado para designar un tipo de organización social en el que la autoridad la ejerce el varón jefe de familia, dueño del patrimonio, del que formaban parte los hijos, la esposa, los esclavos y los bienes, siendo la familia una de las instituciones básicas de este orden social (Fontela, 2008). Entre las estructuras fundantes de este sistema de ordenamiento social se encuentran: 1) el cuidado de los hijos; 2) la heterosexualidad obligatoria; 3) el sistema de propiedad sexual de los hombres sobre las mujeres; 4) El sistema de sexo/género estaría conformado por ideas, normas y convenciones que han llegado a alcanzar el estatuto de leyes naturales sobre la sexualidad y el desarrollo y comportamiento de hombres y mujeres (Rubín, 1986).

Al decir que el género es una construcción, esto no significa que sea una ilusión o una construcción artificial. Se trata más bien de que existe una producción discursiva ${ }^{8}$ que hace aceptable la relación binaria. En ese marco, es que algunas configuraciones culturales del género ocupan el lugar de "lo real", logran auto naturalizarse, y gracias a esto se convierten en hegemónicos en el sistema ( Butler, 1990, p. 99).

De modo que este -el género- está condicionado por normas obligatorias que lo hacen definirse en un sentido, dentro de un marco binario, y por eso la reproducción del género siempre se trata de una negociación de poder. Pero la estructura de dicho mecanismo, ofrece herramientas para socavar su instauración, es un modelo que tiene las bases para desnaturalizarlo. Por eso es que se puede lograr desplazar al género más allá del binarismo naturalizado, apropiarse del género para socavar la estructura misma (Butler 2004; Saxe 2016).

Para Lamas (1997) nacemos dentro de un tejido cultural donde ya están definidas las valoraciones y creencias sobre "lo propio" de los hombres y "lo propio" de las mujeres, es decir, nuestra conciencia ya está habitada por el discurso social, discurso que es el que va estructurando las relaciones sociales de los sexos, por lo que comprender el esquema cultural de género lleva a desentrañar la red de interrelaciones e interacciones sociales del orden simbólico vigente (Lamas, 1997, p. 8).

\subsection{Algunos conceptos teóricos de la Masculinidad}

Estas primeras definiciones nos ayudan a centrarnos en los ámbitos a ser estudiados para comprender la percepción de los y las estudiantes sobre los cambios en actitudes y comportamientos masculinos, siempre y cuando analicemos las diferentes dimensiones $y$ significados de las diferentes masculinidades.

Según Nascimineto (2011) "la masculinidad hegemónica es un modelo que defiende la idea de un hombre fuerte, varonil, profesional, hombre de familia, del mundo público, sin conexión con la esfera de la vida privada, dificultades de transitar por el mundo de los afectos que puede comportarse de manera agresiva (e incluso violenta) contra las mujeres y hombres; convive con representaciones de lo masculino." (Nascimento, 2011, pág. 14-16).

\footnotetext{
${ }^{8}$ El lenguaje, entonces, crea identidades sexuales binarias, fijas y excluyentes que ignoran la fragmentación interna de la clase, el color, la edad, la religión, lo opción sexual, etc. Esas inscripciones del lenguaje son contingentes y crean una fantasía de estabilidad y coherencia con fines de lograr la hegemonía de un modelo y el disciplinamiento de los seres. De acuerdo a Femenías (2006) Butler (2004) se refiere a este tipo de fundamentos cuando habla de la falsa estabilidad de la categoría "mujeres".
} 
De ahí que los hombres que reproducen la masculinidad hegemónica son aquellos hombres que sacan provecho de esa hegemonía para sus intereses. Es por esto que al momento de analizar ámbitos como las masculinidades debemos partir de que un gran porcentaje de estos hombres forma parte del mismo tejido social que reproduce el sistema patriarcal, de modo que fueron educados para ser machistas en todos los ámbitos posibles: familia, escuela, trabajo, entre otras. Entendiendo al machismo como la ideología que engloba el conjunto de actitudes, conductas, prácticas sociales y creencias destinadas a promover la negación de la mujer como sujeto, es decir, hacer pasar por natural que los hombres son más inteligentes, fuertes, líderes natos, dominantes, racionales, cuerdos y capaces en todos los ámbitos (Dimeo, 2015, p. 5).

Para algunos autores los términos "masculinidad tradicional y masculinidad hegemónica" son sinónimos (Connell,1995; Kimmel, 1997; Viveros Vigoya 2003). La primera -M. tradicional- alude a los roles que una sociedad en particular atribuye o espera que sean realizadas por los varones (proveedor, protección, etc.). Mientras que la segunda -la M. hegemónica- define un tipo de relación asimétrica entre hombres y mujeres, y entre los propios hombres.

En este sentido, existe una relación íntima entre masculinidades, hegemonía y subordinación. Hegemonía, al decir de Gramsci (citado por Connell, 2003), se refiere a la dinámica cultural por la cual un grupo exige y sostiene una posición de liderazgo en la vida social, y en cierto grado, con la resignada aceptación de los grupos subordinados, y por subordinación como la dependencia de una persona respecto de otra o de otras, por las que está regida o a las que está sometida. Como se ha descrito en los dos estudios señalados anteriormente, mantener dicho sistema de opresión requiere de prácticas e ideologías basadas en la amenaza y la violencia: sexismo, heterosexismo, homofobia, clasismo, racismo entre otros (Negrete, 2016, p. 7).

Esta multiplicidad de formas de opresión Connell $(1995,2005)$ se corresponde con dos formas de hegemonía: la externa, que hace referencia a la dominación de los hombres sobre las mujeres y la interna, es aquella que se produce entre los propios hombres (Barbero, 2017, p. 11). Pero, a su vez, estas como categorías de estudio estarían modificadas por el contexto cultural, social, económico y político donde ésta se inscribe, y a su vez, atravesada por variables como la edad, etnia, posición social entre otros (Badinter, 1992; Connell, 1998).

En términos generales, podríamos resumir las características de la masculinidad hegemónicatradicional en las siguientes (Dimeo, 2015; Casademont, 2010):

- Exige que el hombre sea el proveedor infalible, que no exprese sentimientos,

- que trate a las mujeres como objetos, que sea sexualmente excelso, incluso que sea violento en su cotidianidad.

- Busca naturalizar la exigencia opresiva de que la mujer efectúe extenuantes jornadas asumiendo que ha nacido con la cualidad de la multitarea.

- Justifica la violencia asumiendo que los hombres son violentos por naturaleza, por lo que son propensos a golpear, violar y someter a las mujeres; de modo que sea responsabilidad de ellas cuidarse del acoso, violaciones y asesinatos recluyéndose al espacio privado del hogar.

- La masculinidad es una forma de complicidad entre varones en la que las mujeres y los homosexuales son los otros; unos otros a quienes se asigna un estatus social inferior (Guasch, 2008, p. 38).

Una vez planteado el marco y matriz teórica conceptual desde la cual serán analizadas las entrevistas se procede a presentar la matriz de análisis, de elaboración propia, al cual se fueron sometidos los datos.

Revista del Laboratorio Iberoamericano para el Estudio Sociohistórico de las Sexualidades https://doi.org/10.46661/relies.5521 


\subsection{Variables y Dimensiones Estudiadas}

Para la recolección de los datos se utilizó como instrumento la entrevista semiestructurada abierta y cerrada acompañada de un cuestionario guía compuesto de 30 preguntas. Las entrevistas se aplicaron a 24 estudiantes (12 varones y 12 mujeres) de edades entre 23 a 45, quienes residían en la ciudad de Asunción y el departamento central, las y los entrevistados corresponden a estudiantes de las carreras de Psicología, Trabajo Social, Derecho y Medicina.

Tabla No. 1 Variables e Indicadores

\begin{tabular}{|c|c|c|c|}
\hline Variables & Operacionalización & Dimensiones & Indicadores \\
\hline \multirow{2}{*}{$\begin{array}{c}\text { Influencia del } \\
\text { sistema patriarcal } \\
\text { en la configuración } \\
\text { de las } \\
\text { masculinidades. }\end{array}$} & \multirow{2}{*}{$\begin{array}{l}\text { La influencia se entiende como los efectos que causan la } \\
\text { división sexual del trabajo, el sistema de ordenamiento } \\
\text { social sobre actitudes y comportamientos de los } \\
\text { estudiantes varones y mujeres en el periodo } 2016 \text {. }\end{array}$} & $\begin{array}{l}\text { Roles de } \\
\text { género }\end{array}$ & $\begin{array}{l}\text { Tareas domésticas } \\
\text { Tareas de cuidado }\end{array}$ \\
\hline & & Masculinidades & $\begin{array}{l}\text { Características } \\
\text { masculinas }\end{array}$ \\
\hline $\begin{array}{l}\text { Cambios en } \\
\text { actitudes y } \\
\text { comportamientos } \\
\text { de los jóvenes }\end{array}$ & $\begin{array}{l}\text { Los roles o tareas caracterizadas como femeninas y } \\
\text { masculinas. } \\
\text { Cambios en comportamientos que contradicen los roles } \\
\text { de géneros tradicionales. }\end{array}$ & $\begin{array}{l}\text { Roles de } \\
\text { género }\end{array}$ & $\begin{array}{l}\text { Tareas domésticas a } \\
\text { cargo de varones } \\
\text { Tareas de cuidado a } \\
\text { cargo de varones } \\
\text { Nueva forma de } \\
\text { relacionamiento }\end{array}$ \\
\hline $\begin{array}{l}\text { Características de } \\
\text { actitudes y } \\
\text { comportamientos } \\
\text { de varones y } \\
\text { mujeres en } \\
\text { sexualidad y las } \\
\text { generaciones } \\
\text { jóvenes. }\end{array}$ & $\begin{array}{l}\text { Refiere al aspecto simbólico de lo que es ser varón o } \\
\text { mujer, así como la conducta y el proceder de cada uno } \\
\text { de los géneros en la sociedad. Esto se expresa a través } \\
\text { de los roles, tareas, cualidades. Los mandatos culturales } \\
\text { en lo que respecta a la sexualidad, lo simbolizan como } \\
\text { natural. Esto depende en gran medida del orden social } \\
\text { que le atribuye cada generación. }\end{array}$ & Sexualidad & $\begin{array}{l}\text { Percepción de lo que } \\
\text { caracteriza a varones y a } \\
\text { mujeres desde la } \\
\text { sexualidad }\end{array}$ \\
\hline $\begin{array}{l}\text { Responsabilidades } \\
\text { que asumen } \\
\text { estudiantes varones } \\
\text { y mujeres en la } \\
\text { salud reproductiva }\end{array}$ & $\begin{array}{l}\text { El compromiso u obligación que cada individuo } \\
\text { reconoce, independientemente de su sexo género. Para } \\
\text { tomar o no decisiones en lo que respecta a su cuerpo de } \\
\text { manera autónoma y consciente }\end{array}$ & $\begin{array}{c}\text { Salud } \\
\text { reproductiva }\end{array}$ & $\begin{array}{l}\text { Sobre quien recae la } \\
\text { Responsabilidad de la } \\
\text { salud reproductiva; } \\
\text { varones } \\
\text { mujeres } \\
\text { ambos }\end{array}$ \\
\hline $\begin{array}{l}\text { Percepción de } \\
\text { estudiantes sobre la } \\
\text { paternidad. }\end{array}$ & $\begin{array}{l}\text { Las ideas e impresiones que tienen sobre lo que implica } \\
\text { ser padre; su importancia o no durante el desarrollo de } \\
\text { vida de los niños y niñas. }\end{array}$ & Paternidad & $\begin{array}{l}\text { Percepción sobre: } \\
\text { La crianza o educación de } \\
\text { los hijos/as } \\
\text { La presencia paterna para } \\
\text { el desarrollo de los niños } \\
\text { y niñas }\end{array}$ \\
\hline $\begin{array}{l}\text { Comportamientos } \\
\text { masculinos } \\
\text { tradicionales que } \\
\text { propician la violencia } \\
\text { física y psicológica } \\
\text { entre varones y } \\
\text { mujeres }\end{array}$ & $\begin{array}{l}\text { La conducta del varón tradicional que acepta la violencia } \\
\text { como mecanismo compensatorio para solucionar } \\
\text { diferencias y afirmar el poder y control } \\
\text { La violencia de los varones es resultado de su poder, de } \\
\text { privilegios, del permiso para ejercerla y del temor de } \\
\text { carecer del poder }\end{array}$ & Violencia & $\begin{array}{c}\text { Física } \\
\text { Psicológica } \\
\text { Económica } \\
\text { Generacional }\end{array}$ \\
\hline
\end{tabular}




\subsection{Diseño Metodológico}

Como técnica se utilizó la entrevista semiestructurada abierta y cerrada, para lo cual se elaboró un cuestionario con 30 preguntas guías, dichas preguntas se dividen en cinco las cinco dimensiones, especificadas en la tabla anterior con sus indicadores pertinentes. El cuestionario fue elaborado con ayuda de una especialista en Género y Violencia de Paraguay.

La población estuvo representada por estudiantes de los últimos años de cuatro facultades de la UNA de la Capital de Asunción del Paraguay. El diseño metodológico, es de tipo exploratorio. En cuanto a las variables y dimensiones estas se dividen en 6: la influencia del sistema patriarcal (Roles de género, 1) en las actitudes y comportamientos de las nuevas generaciones de jóvenes (2). Calculados a partir de indicadores como características de comportamientos y actitudes de varones y mujeres en sexualidad (3), la salud reproductiva (4), la percepción de estudiantes sobre la paternidad (5) y los comportamientos que propician la violencia física (6) y psicológica entre varones y mujeres

Para la recolección de datos se solicitó autorización mediante nota a las Facultades, de las cuales sólo la Facultad de Filosofía y el Instituto de Trabajo social autorizaron la recolección de datos. No obstante, debido a la demora por parte de las autoridades académicas se prefirió entrevistar a los estudiantes en los centros de prácticas y fuera de las universidades, en lugares como cafetería y oficinas.

La recolección de datos se realizó en 4 semanas: desde mediados de julio/2016 hasta la primera semana de agosto/2016. Como se mencionó la selección fue aleatoria y una vez explicada la finalidad de la entrevista los y las estudiantes deciden participar de forma voluntaria, también fueron consultados/as sobre la utilización de la grabadora como instrumento, al que en su mayoría accedió, ya que cada entrevista duró aproximadamente entre 20 a 25 minutos.

Posteriormente se procedió a clasificar las entrevistas por carreras y género en una planilla de Microsoft Excel. Para la transcripción se clasificó cada respuesta teniendo en cuenta la relación existente entre ellas, el tiempo que tomó la clasificación fue de 4 semanas aproximadamente.

Entre las limitaciones existentes para la investigación se encuentra: la burocracia administrativa por el tiempo que tardaron las notas de permiso de las facultades. La falta de perspectiva de género del entonces director académico para la aprobación del proyecto, y los pocos recursos con los que se disponía para el estudio de grado.

Las consideraciones éticas tomadas para el estudio fueron: la confidencialidad de los datos brindados por parte de los y las participantes para resguardo de integridad personal. El cuestionario fue de carácter investigativo, es por ello que para el análisis de los datos se utilizó la sigla del primer nombre y el primer apellido. Los datos obtenidos son exclusivamente con fines académicos, los criterios de inclusión no tienen efectos en la población seleccionada para el estudio. 


\section{Análisis de datos y resultados}

En esta sección se presentan el análisis de la información obtenida y los principales resultados del estudio mediante algunos gráficos referentes a la distribución de las tareas domésticas:

Gráfico No. 1 Ocupación de Tareas Domésticas

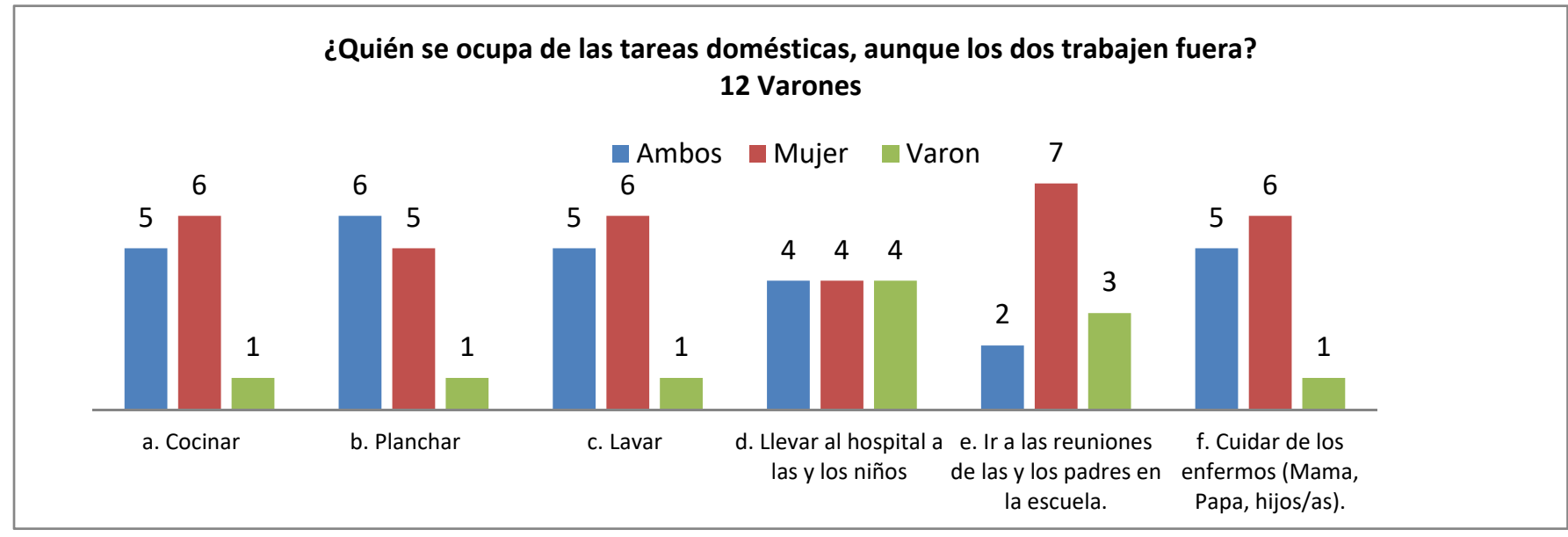

Como se puede observar de un total de 12 varones entrevistados, los datos arrojados presentan una aparente distribución de las tareas. Sin embargo, sobre tareas como cocinar, lavar y cuidar a los enfermos 6 de los doce entrevistados refieren que esa tarea es de las mujeres. Es decir que, la mitad de las 24 entrevistas mencionan que las tareas domésticas son todavía consideradas femeninas, en cambio existe otro grupo no mayor de estudiantes que coinciden en que las tareas domésticas hoy día las realizan ambos por igual.

Gráfico No. 2 Distribución de tareas por Género

1.1. Varones - Mujeres

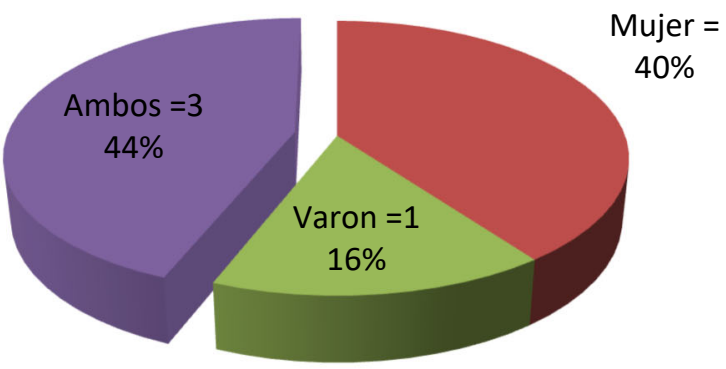

Una de las preguntas planteaba la interrogante de 1.3 ¿Crees que los hombres son capaces de cuidar a sus hijos? De las 24 personas entrevistadas, un total de 22 consideran que los varones son capaces de cuidar a los hijos e hijas seguidas de dos personas que refieren que no. Seguidamente en la pregunta 1.4 los entrevistados y entrevistadas debían citar las características con las que debe contar un varón para ser considerado "bien hombre".

Entre las características con las que un varón debe contar para ser considerado "bien hombre" las más citadas por varones se relacionan a rasgos sexuales biológico como genitales y el órgano reproductor masculino, la fuerza, y características secundarias y rasgos propios de la expresión de género, como la forma de vestir, entre otras cualidades. Las mujeres a modo de caracterizar al sujeto 
masculino, también refieren al hecho de ser un hombre trabajador y por ende responsable del hogar y de la seguridad de las familias.

Gráfico No. 3 Características Masculinas

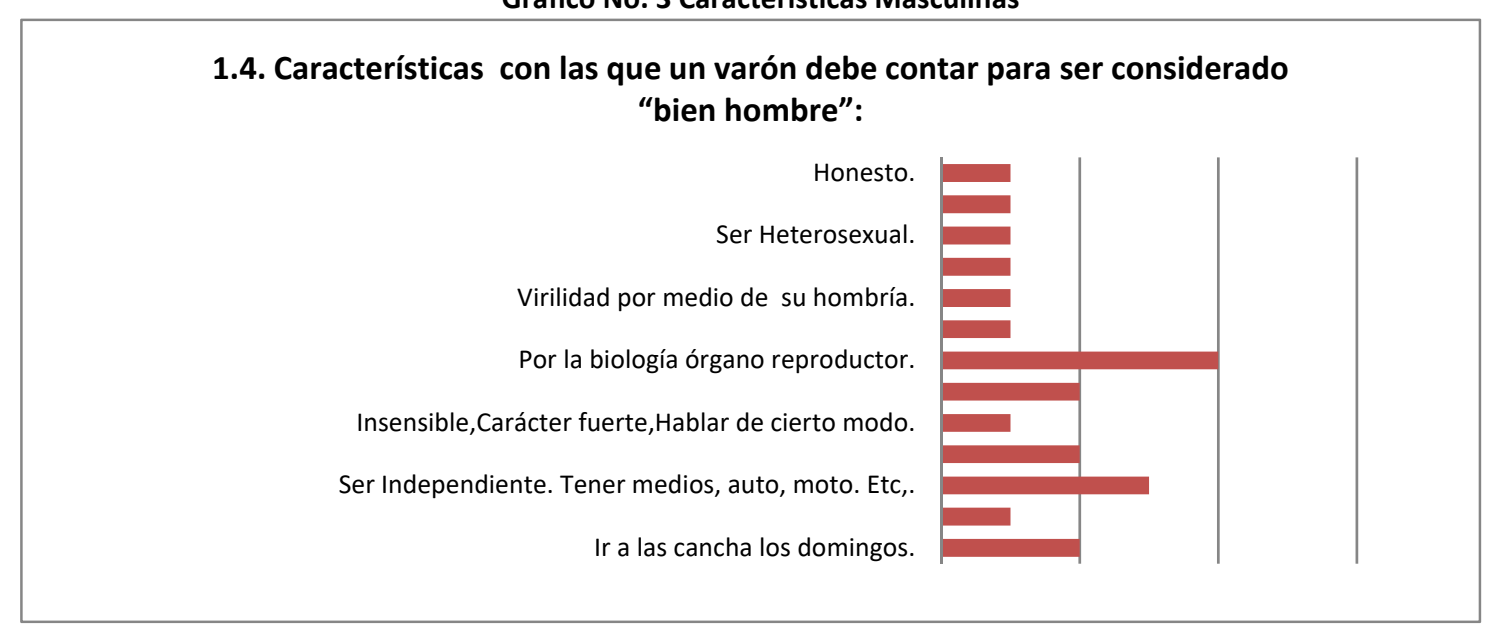

"Deber ser atento, ir a la cancha los domingos". "El rostro y la musculatura". (Ev 1 psicología, 40 años).

"Para mí; no existen características específicas con las que debe contar, solo debe tener un cromosoma más y uno menos". (Ev. 7 medicina, 22 años).

"Que sea bruto. Que tenga iniciativa. Exprese sus emociones. Trabajo estable". (Ev. 5 psicología, 22 años).

"Se debe encargar de la parte económica. Tener el pelo corto. No tanto esmero en lo personal. (Ev. 10 medicina, 25 años).

\subsection{Masculinidad}

Ahora bien, si vamos a la dimensión de Masculinidad, 6 -de 12- mujeres entrevistadas afirman que los varones son rudos, fuertes y ágiles por su condición masculina y condición biológica. En cambio, los varones las contradicen afirmando que el género femenino ha demostrado que ambos pueden hacer las mismas cosas. Dicha contradicción está relacionada a dos cuestiones: la primera, al empoderamiento de las mujeres y la conquista de espacios en escenarios antes considerados masculinos producto de las demandas de los movimientos feministas, y la segunda estaría relacionada con las características biológicas y culturales propias de la masculinidad.

"Podría ser porque hay cosas que yo no puedo hacer qué puede hacer mi marido o mi hermano. "Cargarle a mi mamá". (Em. 17 derecho, 32 años).

"Cada día las mujeres están demostrando que pueden realizar las mismas actividades que los hombres". (Ev.13 Derecho, 25 años).

"No tiene que ser fuertes o ágiles por ser masculinos; también la mujer puede ser ruda y tener esas características". (Em. 20 T. Social, 27 años).

"Hay hombres que son femeninos". (Ev. 24 T. Social, 28 años).

Para los varones la represión de sus emociones y sentimientos, es una de las maneras en la cual el niño varón debe reafirmar su masculinidad, es decir que, a la primera señal de debilidad, el niño es

\footnotetext{
${ }^{9}$ Parte de las entrevistas consistía en argumentar ¿Por qué? de sus respuestas y estas serán abreviadas en EV si son varones y EM si son mujeres.
}

Revista del Laboratorio 
inducido, generalmente por sus pares, a reprimir emociones debido al género al que pertenece, este no debe demostrar actitudes que son consideradas expresiones femeninas.

Continuando con las dimensiones de la Masculinidad se interrogó a los y las estudiantes quien en una relación de pareja tiene la última palabra (ver 2.3). Respecto a los varones se puede observar que en su mayoría refieren que el género masculino, mientras que menos de la mitad refiere que ambos. En el transcurrir de las entrevistas se puede observar cómo el discurso es bastante contradictorio a la hora de ejercer un rol dentro de las relaciones personales.

Gráfico No. 4 Toma de decisiones en la Relación

2.3. En una relación de pareja entre un hombre y una mujer ¿quién tiene la última palabra?

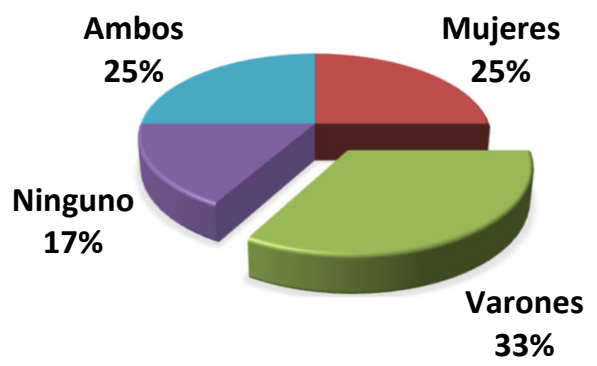

Las respuestas atribuyen un mayor nivel de responsabilidad a las mujeres en las relaciones varónmujer; la masculinidad tradicional engloba las conductas y actitudes con las que debe contar los varones, y la responsabilidad no es una de ella. La independencia masculina conlleva una serie de comportamientos que generalmente llegan a extremos, desde los cuales asumir las consecuencias de actos no está en ella.

Posteriormente se presentó la interrogante 2.4 ¿Crees que hoy día las actitudes y comportamientos de los varones van cambiando hacia nuevas formas de ser masculinos, comparadas a lo que se considera tradicional? ¿Por qué? La totalidad de los entrevistados (24) refiere que sí, y entre los argumentos se encuentran:

$\checkmark$ Mayor apertura mental tanto del varón como la mujer.

$\checkmark$ Hoy en día se incentiva la igualdad de género.

$\checkmark$ Los roles se van equiparando más, la brecha se va acortando.

$\checkmark$ Los varones como se visten por ejemplo un tipo que usa camisa rosada no necesariamente es gay.

$\checkmark$ Hay varones que se dedican a la cocina y al baile.

$\checkmark$ Se van modificando pasando a darse de forma democrática

$\checkmark$ Esto se debe a la necesidad de hoy día;

$\checkmark$ Se están adaptando a nuevas formas de vida, el empleo informal, la mujer sale y el hombre se queda en la casa.

$\checkmark$ Los nuevos padres tienen otra mentalidad, una nueva construcción.

Manifestaron que existe una transformación en lo que se consideraba al varón tradicional; para algunos esto se debe a la lucha que existe por igualdad de los géneros, en cambio otros manifiestan que el cambio se da de manera más superficial refiriendo a que hoy día los varones utilizan prendas,

Revista del Laboratorio Iberoamericano para el Estudio Sociohistórico de las Sexualidades https://doi.org/10.46661/relies.5521 
colores, accesorios y realizan labores, deportes que antes eran considerados femeninos. Entonces tanto varones y mujeres en mayor medida coinciden en que los cambios apuntan a una mayor apertura o la incursión de estos, así como mujeres en deportes, el mundo laboral o la moda.

Ambos, varones y mujeres refieren que la masculinidad tradicional incide negativamente para el surgimiento de otros modelos de masculinidad. Un total de 18 personas la calificó de manera negativa, algunos atribuyen el cambio o la transformación de la masculinidad tradicional a la situación del sistema de mercado laboral, ya que la demanda de fuerza de trabajo obliga al sistema económico a exigir mayor producción a menos costos insertando a las mujeres, niños, niñas y adolescentes, a las leyes de oferta demanda y a la flexibilización.

Otros apuntan a que existe una resistencia al cambio, ya que romper con las viejas normas no generan privilegios para los varones, en cambio otros mencionan que las viejas jerarquías o normas se transforman y estas propician a que capacidades de los varones antes no desarrolladas o adquiridas hoy día los fortalezca como ser humano.

\subsection{Responsabilidad, sexualidad y salud reproductiva}

Con relación a la sexualidad se pidió a las y los participantes que respondan con Sí o NO la siguiente afirmación: ¿Crees que los varones necesitan tener más sexo que las mujeres? A los que un total de 67 por ciento de participantes dijo que SI.

Gráfico No. 5 los varones necesitan tener más sexo que las mujeres

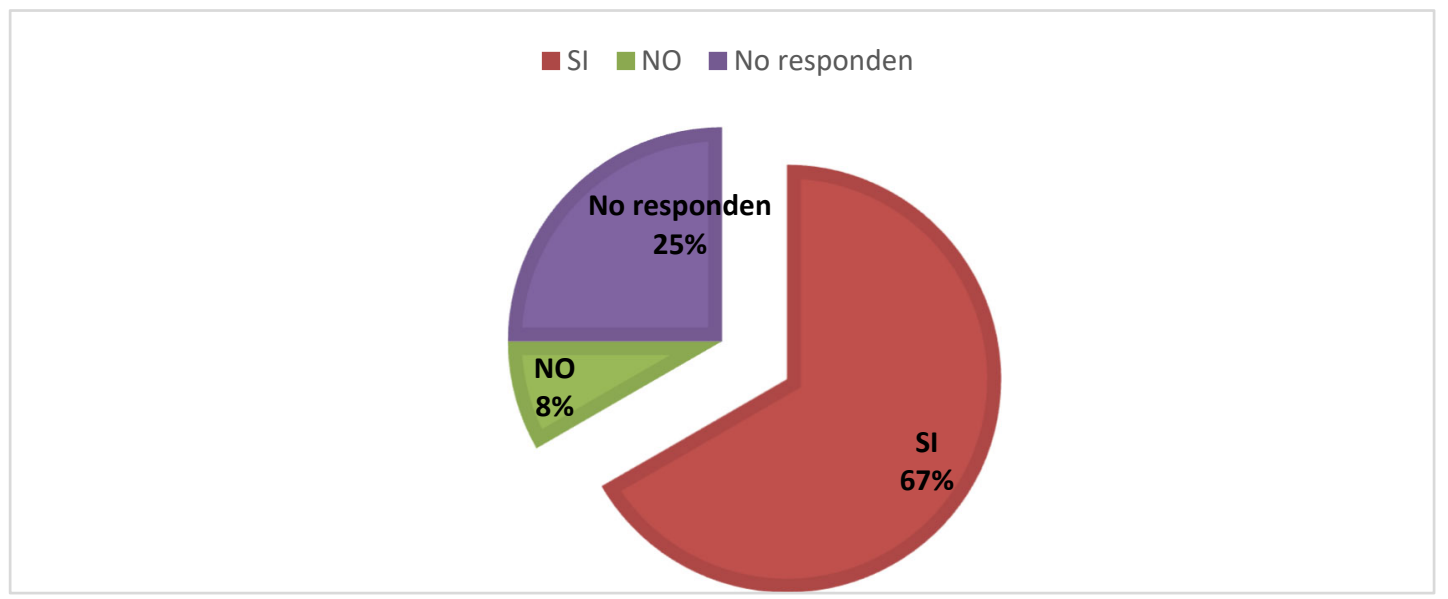

No obstante, al ser interrogados del porqué de dicha respuesta manifestaron cuestiones como "Hay una disposición del mismo organismo debido a que es más activo" "No es una necesidad es algo que hoy en día se da más hincapié" "Tiene que ver mucho con el machismo porque cuando más hombre sos, tenés más relaciones, más mujeres, desde ese punto si es machista".

Se puede observar que, a pesar de la apertura a ciertos cambios como la responsabilidad de las tareas domésticas, el cuidado de los hijos y el empoderamiento femenino dichas ideas se ven contradichas en el campo de la sexualidad y las relaciones de poder. Es decir, el hecho de que se siga afirmando la necesidad del hombre por su condición biológica confirma la existencia de mandatos sociales para con los varones.

Muchos concuerdan con que los instintos masculinos no son sólo una cuestión natural, sino que existen factores que propician a que estos actúen así, otras personas por ejemplo creen que el nivel educativo también influye para estas actitudes y comportamientos. Por ejemplo, en su mayoría han sido los hombres quienes atribuyen sus deseos a un impulso natural. Al contrario de las mujeres se presuponen más, selectivas, prudentes 0 , en otros términos, limitadas por el condicionamiento social y hasta biológico para tener relaciones sexuales.

Revista del Laboratorio Iberoamericano para el Estudio

Sociohistórico de las Sexualidades https://doi.org/10.46661/relies.5521 
"Debido a que hay una mayor predisposición orgánica del varón para realizar eso". (Ev. 1 psicología, 40 años).

"Es genético tiende a ser más impulsivo. La mujer es como que controlan algo más". (Em.

11 medicina, 24 años).

"El hombre que está disponible para las mujeres es más hombre. Y las mujeres son las que deciden cuando quieren tener". (Ev. 1 psicología, 40 años).

Lo antes señalado obedece a que de un total de 24 participantes fueron 21 las personas que afirmaron que el uso del preservativo es responsabilidad de la mujer, mientras que sólo un hombre refirió como responsable a los varones, y, una mujer que manifestó que la responsabilidad debería ser de ambos. Es decir que, la responsabilidad de la prevención de ITS, o embarazo es única y exclusivamente de las mujeres pues lo "común o socialmente aceptable" es que está (el sujeto femenino) asuma los roles de cuidado y protección mientras que los varones deben ser irresponsables, poco prevenidos, e insensible hacia las necesidades demás y su entorno "por su predisposición biológica".

"La mujer, tendría que ver cómo es la que tiene más cabeza, ya que se encarga de las cosas de la casa entonces es ella la que está pendiente sobre su cuerpo, a veces dudo si es para no tener tantos hijos o prevenir enfermedades como que tampoco se discute mucho". (Em. 20 T. Social 27 años).

Gráfico No.5 Salud Reproductiva

\section{1. ¿Por lo general quien es el que propone el uso del preservativo al momento de las relaciones sexuales?}

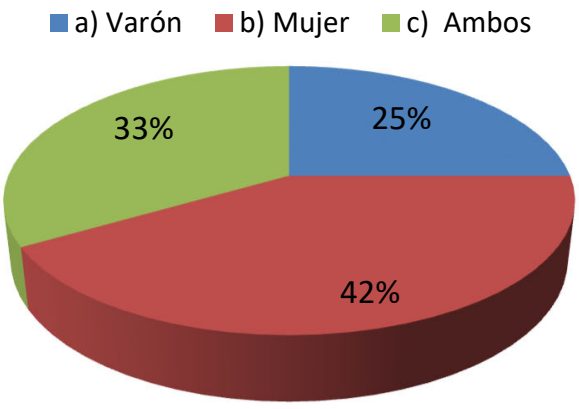

\subsection{Paternidad}

Como última dimensión sobre la paternidad, los datos arrojan que, en el país, la madre es la principal responsable de la crianza de los hijos e hijas; algunas personas afirman que en caso contrario son los abuelos, pocas veces se involucra al padre. Respecto a la importancia de una presencia paterna para el desarrollo de los niños y niñas fueron 23 los estudiantes que manifiestan como importante la presencia del padre. Es decir, tanto para varones como para mujeres las figuras femeninas y masculinas como modelos para los niños eran de gran importancia.

"El hijo tiene el derecho de que esté el padre, además de que las investigaciones hablan que es importante además de la disciplina". (Em. 2 psicología 24 años).

"Yo me crié mayoritariamente con mujeres y creo que salí bien, no sé. Creo que es importante que en el momento que se está criando vea una persona físicamente como vos". (Ev. 14 derecho 23 años).

"La figura paterna y materna influye de manera positiva". (Ev12. Medicina 24 años).

"No creo que sea necesario; si el hombre no quiere asumir la paternidad no podemos obligarlo porque es una decisión de la mujer cuando y con quien quiere tener hijos no hace falta que el hombre 
esté" (Em. 23 T. Social 31 años).

Y, para finalizar, sobre los tipos de violencia los entrevistados y entrevistadas (24) concuerdan que son los varones quienes utilizan la violencia física en una relación de pareja, otras coinciden en que los varones ven a su pareja como parte de su propiedad. Podría deberse a que los estándares de educación con base a los cuales son construidos las categorías de varón-mujer dan legitimidad a las normas y prácticas que se vinculan a los mandatos de masculinidad hegemónica como fue mencionado en el segundo escenario.

En este punto, las personas entrevistadas debían opinar sobre una situación, la misma consistía en que el hombre o varón reacciona violentamente en contra de su pareja debido a que ella no lo obedece. Ambos grupos coinciden que la violencia no es la manera de resolver las diferencias, y que los varones que actúan de esa manera están mal y que se deben buscar soluciones razonables a tales ideas que atentan contra la integridad y derecho de todos y todas.

Pero al invertir la situación, siendo un hombre víctima de la violencia los entrevistados refieren que no denunciaron el hecho en caso de ser agredidos o violentados por su pareja. Esto se debería, como hemos visto más arriba, a los mandatos de masculinidad existentes a nivel cultural, social y simbólico. Sobre todo, en lo que respecta a los pares varones, no cumplir con los mandamientos de lo que significa ser un hombre conlleva una carga de burlas, humillaciones y más violencia por parte de otros varones.

\section{Reflexiones Finales}

Entre los resultados se destaca que estudiantes, tanto varones y mujeres, identifican y perciben la influencia del sistema de la división sexual del trabajo a través de las tareas domésticas y mediante los mandatos del binarismo genérico imperante en la sociedad paraguaya. En lo que respecta a actitudes y comportamientos considerados negativos sobre los roles de géneros de cada individuo se concluyó que reproducir actitudes y comportamientos machistas es más frecuente en los varones. Dicha conclusión se da luego de constatar que los comportamientos discriminatorios y sexistas, como el cuidado y la salud reproductiva siguen siendo exclusiva responsabilidad de las mujeres, situación que reafirma que la influencia negativa de los roles tradicionales de género sin duda trasciende el nivel académico, profesional, político y social.

A esto se agrega que las personas encuestadas mediante un discurso argumentativo entienden y aseguran que las tareas de la casa son responsabilidad de ambos -varones y mujeres- pero en la práctica esto no es así, ya que al profundizar sobre las experiencias -o las tareas que mencionan realizar- estas no concuerdan con lo que opinan sobre la realidad.

Con respecto a la masculinidad, aspectos como: demostrar sentimientos, tomar decisiones con responsabilidad y las implicancias del modelo tradicional reflejan que existe un cambio dentro de la masculinidad propiamente dicha, pero que la masculinidad tradicional mediante sanciones sociales incide negativamente tanto en varones como en mujeres, en vista que las formas de expresión y relacionamiento permitidas por la masculinidad hegemónica afectan ámbitos bio-psico-sociales de las personas, por ejemplo: la conducta, el tipo de relación social con otras personas, la limitación emocional para el manejo de sentimientos, lo que dificulta la solución de conflictos de forma razonable.

En este punto es importante resaltar que las conclusiones de este estudio debido a sus características no son generalizables, aunque en él se abordan aspectos de relevancia social sobre todo debido al aumento de casos de feminicidios en el país y la responsabilidad social que poseen las universidades y los centros educativos en la reproducción de patrones conductuales. No obstante, es reseñable como en cierto momento las y los participantes refieren que en sus 
experiencias existen cambios en los varones relacionados con la demostración de afecto, la incursión en campos antes calificados como femeninos como la moda, la cocina, el baile y etcétera. Pero, pareciera que dichos cambios son meramente superficiales.

En relación a las características entre varones y mujeres en la sexualidad: ambos grupos refieren que los varones necesitan tener más sexo que las mujeres argumentando que esto se debe a los impulsos masculinos. Es decir, se califica a los varones como "aquellos que utilizan su condición (masculina) para actuar de formas que, para otras y otros sujetos, no están permitidas socialmente". El argumento más mencionado por las y los participantes es el que los varones son impulsivos debido a su "naturaleza", obviando que, en la mayoría de las veces, el ser humano no solo responde a instintos sino además a mandatos culturalmente establecidos.

En cuanto a la paternidad casi siempre son las madres quienes asumen la responsabilidad y la crianza de los hijos e hijas, cuenten o no con el apoyo de los varones. Sin embargo, la totalidad de las entrevistas arrojaron la importancia de la presencia paterna en el desarrollo armónico biopsicosocial del niño niña y adolescente, siempre y cuando se trate de varones que lo hacen responsablemente y acompañan el crecimiento y desarrollo de sus capacidades, fomentando una masculinidad que respete la integridad de las demás personas y seres vivos. Además, se menciona mayoritariamente al rol paterno con el de proveedor de la casa y seguridad, esas son las funciones paternas mencionadas repetitivamente por los y las estudiantes.

En relación a los comportamientos y actitudes que propician la violencia se concluye que, en la mayoría de los casos, es producto de mandatos culturales que ciertamente justifican la violencia en todos sus ámbitos como medio para entablar relaciones sociales tal y como pudo observar en los tres escenarios descritos en el apartado dos. Es decir, aún se siguen justificando comportamientos que atentan contra la integridad física y psíquica de otras personas, bajo la excusa de la "condición biológica" des-responsabilizando a la sociedad, en general y a los individuos, en particular, de las diferentes formas de violencia, que son productos y consecuencias de un proceso del que, de manera consciente o no, todos y todas formamos parte. 


\section{Bibliografía}

Aguayo, F; Correa, P; Cristi, P. (2011). Encuesta IMAGES Chile Resultados de la Encuesta Internacional de Masculinidades y Equidad de Género. Santiago: CulturaSalud/EME. Disponible en: https://promundoglobal.org/wp-content/uploads/2015/01/Encuesta-IMAGES-Chile.pdf

Barker, G; Aguayo, F; Correa, P. (2013). Comprendiendo el ejercicio de violencia de los hombres hacia las mujeres. Algunos resultados de la encuesta IMAGES (The International Men and Gender Equality Survey) en Brasil, Chile y México. Rio de Janeiro: Instituto Promundo. Disponible en: https://www.paho.org/hq/dmdocuments/2014/Comprendiendo-el-ejercicio-de-violencia-dehombres.pdf

Barbero, M. D. S. (2017). Hacerse hombre en el aula: masculinidad, homofobia y acoso escolar. cadernos pagu, (50). Disponible en: https://www.scielo.br/pdf/cpa/n50/1809-4449-cpa18094449201700500014.pdf

Connell, R. (1995) Masculinidades. México, PUEG, 1995.

Connell, R. (2005) Gender. Cambridge, Polity Press, 2002. CONNELL, R.W.; Messerschmidt, J.W. Hegemonic masculinity: Rethinking the concept. Gender and Society, vol. 19, no 6, dec. 2005, pp.829- 859 .

Dimeo, Mauricio. (2015) Porque una Masculinidad Disidente. Disponible en https://altersexual.net/2015/09/10/por-que-una-masculinidad- disidente-por-mauricio-dimeo/

Fontenla, M. (2008) "Diccionario de estudios de Género y Feminismos". Editorial Biblos. Disponible en: http://www.mujeresenred.net/spip.php?article1396

Guasch, A. O. (2008). Los varones en perspectiva de género. Teorías y experiencias de discriminación. Asparkía: investigació feminista, 29-38. Disponible en: https://tinyurl.com/y5nkl24d

Guasch, A. O. (2007). ¿Por qué los varones son discriminados por serlo?: masculinidades, heroísmos, y discriminaciones de género. In Cultura, homosexualidad y homofobia (pp. 87-104). Editorial Laertes.

Guevara Ruiseñor, E. S. (2006). Construcción de la masculinidad en la escuela y la familia en jóvenes universitarios. Psicología para América Latina, (8), 0-0. Disponible en: http://pepsic.bvsalud.org/scielo.php?script=sci arttext\&pid=S1870-

350X2006000400015http://pepsic.bvsalud.org/scielo.php?script=sci arttext\&pid=S1870350X2006000400015

Kaufman, M. (1987). The construction of masculinity and the triad of men's violence. Beyond patriarchy: Essays by men on pleasure, power, and change, 1-29.

Kollontai, A. (1976). La mujer en el desarrollo social. Guadarrama. Disponible en: https://books.openedition.org/ariadnaediciones/1944?lang=es

Kimmel, M. (1997). Homofobia, temor, vergüenza y silencio en la identidad masculina. Masculinidad/es. Poder y crisis, 24, 49-63.

Lamas, M. (2007). El género es cultura. V Campus de Cooperación Cultural. http://www.paginaspersonales.unam.mx/app/webroot/files/981/El genero es cultura Martha L amas.pdf

Nascimento, M. (2015). Improbables Encuentros: un estudio sobre la Masculinidad en el contexto de relaciones de Amistad entre homo y heterosexuales en Brasil. Disponible en: https://tinyurl.com/y6kyql4a 
Negrete, M. (2016) Discursos relacionados a las masculinidades y la violencia en adolescentes escolarizados de Asunción. CLASCO. Nuevas problemáticas de Género y desigualdad en américa latina y el caribe, 93.

Disponible en: http://biblioteca.clacso.edu.ar/clacso/becas/20160401125212/informe

Pérez-Bustos, T., Vigoya, M. V., Lesmes, S., Mera, M. E. G., Ramírez, O. A. Q., Pisano, P., ... Castañeda, O. J. M. Presentación 7.

Disponible en:

http://www.scielo.org.co/scielo.php?script=sci arttext\&pid=S0123-885X2014000200001

Pichardo, J. I. (2012). El estigma hacia personas lesbianas, gays, bisexuales y transexuales. GAVIRIA, E.; GARCÍA-AEL, C.; MOLERO, F.(coords.) Investigación-acción. Aportaciones de la investigación a la reducción del estigma. Madrid, Sanz y Torres, 111-125.

Rodríguez-Covarrubias, M. C. Juárez-Armendáriz, L., Juárez-Ramírez, Y. y López-Padilla, M. A. (2016). La masculinidad y la violencia en jóvenes universitarios. El caso de la FTS de la Universidad Autónoma de Coahuila. Posibilidades y Retos para la 64 . Disponible en: http://ecorfan.org/handbooks/Ciencias\%20Estudios\%20de\%20Genero\%20T-II/8.pdf

Rubio, M. I. (2001). El estudio sobre las masculinidades. Panorámica general. Disponible en: http://www.ugr.es/ pwlac/G17 27Marialsabel Jociles Rubio.html

Rubín, G. (1989). Reflexionando sobre el sexo: notas para una teoría radical de la sexualidad. Placer y peligro. Explorando la sexualidad femenina, 113, 190. 